\title{
Transient growth of Kelvin waves on quantized vortices
}

\author{
C. F. Barenghi ${ }^{1}$, M. Tsubota ${ }^{2}$, A. Mitani ${ }^{2}$ and T. Araki ${ }^{2}$ \\ ${ }^{1}$ School of Mathematics and Statistics, University of Newcastle, Newcastle, NE1 7RU, UK \\ ${ }^{2}$ Physics Department, Faculty of Science, Osaka City University, Japan
}

Quantized vortex lines in helium II can be destabilized by a sufficiently large normal fluid velocity which is parallel to the vortex lines (Donnelly - Glaberson instability). We study what happens if the driving normal fluid is not steady but oscillates periodically with time. We show that in certain situations, although individual perturbations decay for $t \rightarrow \infty$, a state of transient growth of Kelvin waves is possible, which may explain observations of a small and not reproducible longitudinal component of mutual friction.

\section{Introduction}

Recent work on the hydrodynamics of helium II has highlighted the importance of the Donnelly - Glaberson instability. The instability takes the form of Kelvin waves (rotating helical displacements of the vortex core) which grow exponentially along quantized vortex lines. If this happens, the length of the vortex lines increases and energy is transferred from the normal fluid to the superfluid $\mathbb{1}$. The Donnelly - Glaberson instability was discovered ${ }^{233}$ when a small heat current was applied in the direction parallel to quantized vortices in rotating helium II. Figure 1 shows this rotating counterflow configuration. A channel is closed at one end and open to the helium bath at the opposite end. At the closed end a resistor $R$ dissipates a known heat flux $Q$. The heat is carried away from the resistor by the normal fluid whilst the superfluid flows in the opposite direction to assure that the net mass flux is zero. In this way a counterflow velocity $V$ is set up which is proportional to the applied heat flux. If the channel is rotated about its axis at angular velocity $\Omega$, in the absence of heat flux there would be a uniform array of superfluid vortices of areal density $L=2 \Omega / \Gamma$ aligned along the rotation axis where $\Gamma$ is the quantum of circulation. In the presence 


\section{Barenghi, Tsubota, Mitani and Araki}

of a heat flux, the normal fluid velocity $\mathbf{v}_{n}$ is parallel to the vortices and, if $Q$ is large enough, the Donnelly - Glaberson instability takes place and Kelvin waves grow exponentially with time. When the amplitude of the Kelvin waves becomes of the order of the intervortex spacing $b \approx L^{-1 / 2}$, vortex reconnections take place and, according to a recent calculation ${ }^{4}$, the uniform array of vortices may turn into a state of polarized turbulence.

It must be stressed that, although the rotating heat transfer configuration of Figure 1 is the best to study the Donnelly - Glaberson instability in a controlled way, there are other experiments in which the Donnelly Glaberson instability plays a key role. For example, if helium II is made turbulent using a towed grid 5 or rotating propellers ${ }^{6}$, both normal fluid and superfluid are turbulent (the former consisting of eddies of various sizes and strengths, the latter consisting of a disordered tangle of quantized vortices). In this case the instability may take place throughout the turbulent flow, provided that the component of $\mathbf{v}_{n}$ locally parallel to a superfluid vortex filament exceeds a critical value 78 .

The aim of this paper is to remark that a similar Donnelly - Glaberson instability can be excited if $\mathbf{v}_{n}$ is not steady but varies harmonically with time. The natural way to do this is to modify the configuration of Figure 1 and connect the resistor $R$ to an AC (rather than a DC) power supply. For the sake of brevity we shall refer to the old and new configurations as the "DC instability" and the "AC instability" respectively.

The plan of the paper is the following: after deriving the DC instability in Section 2, we describe two versions of the new instability in Sections 3 and 4. The results are discussed in Section 5 in view of previous experiments.

\section{The DC instability}

Let us consider a quantized vortex which points in the $z$ direction and assume that its shape is perturbed in the form of a small amplitude Kelvin wave of wavelength $\lambda$. A point on the vortex has Cartesian components

$$
\mathbf{s}(\xi)=[A \cos (\phi) ; A \sin (\phi) ; \xi]
$$

where $\phi=k \xi-\omega t, t$ is time, and $\omega, A$ and $k=2 \pi / \lambda$ are respectively the angular frequency, the amplitude and the wavenumber of the wave. Assuming $A k<<1$, it is easy to verify that the parameter $\xi$ is the arc length. In fact $\mathbf{s}^{\prime}=d \mathbf{s} / d \xi=[-k A \sin (\phi) ; k A \cos (\phi) ; 1]$ is the tangent to the curve at the point $\mathbf{s}$ and the length $\Lambda$ measured along the curve is

$$
\Lambda=\int_{0}^{\xi} d \xi_{0} \sqrt{\left|\mathbf{s}^{\prime}\left(\xi_{0}\right) \cdot \mathbf{s}^{\prime}\left(\xi_{0}\right)\right|}=\int_{0}^{\xi} d \xi_{0} \sqrt{k^{2} A^{2}+1} \approx \xi .
$$




\section{Transient growth of Kelvin waves on quantized vortices}

In the absence of friction and using the local induction approximation 9 , the self-induced velocity of the vortex at the point $\mathbf{s}$ is

$$
\frac{d \mathbf{s}}{d t}=\mathbf{v}_{\text {self }}=\nu_{s} \mathbf{s}^{\prime} \times \mathbf{s}^{\prime \prime},
$$

where $\nu_{s}=(\Gamma /(4 \pi)) \log (b / a)$ and $a$ is the vortex core radius. From (11) and (3) we obtain the dispersion relation of Kelvin waves

$$
\omega \approx \nu_{s} k^{2}
$$

In the presence of friction the equation of motion is 10

$$
\frac{d \mathbf{s}}{d t}=\mathbf{v}_{\text {self }}+\alpha \mathbf{s}^{\prime} \times\left(\mathbf{v}_{n}-\mathbf{v}_{s}-\mathbf{v}_{\text {self }}\right)
$$

where $\alpha$ is a known mutual friction coefficient and $\mathbf{v}_{n}$ and $\mathbf{v}_{s}$ are imposed normal fluid and superfluid velocity fields. Choosing the $z$ axis along the counterflow channel as in Figure 1, we have $\mathbf{v}_{n}-\mathbf{v}_{s}=[0 ; 0 ; V]$ where $V$ is the applied counterflow velocity. Allowing the amplitude of the wave to change with time and using (4) we obtain

$$
\frac{d A}{d t}=\alpha\left(k V-\nu_{s} k^{2}\right) A,
$$

If $V$ is constant the solution of equation (6) is

$$
A(t)=A(0) \exp (\sigma t)
$$

where $A(0)$ is the initial infinitesimal amplitude of the wave due to thermal or mechanical vibrations and $\sigma=\alpha\left(k V-\nu_{s} k^{2}\right)$ is the growth rate. The instability occurs if $\sigma>0$. Therefore a Kelvin wave of wavenumber $k$ is unstable in the region $0<k<k_{0}$ where $k_{0}=V / \nu_{s}$ and the maximum growth rate $\sigma_{c}=\alpha V^{2} /\left(4 \nu_{s}\right)$ occurs at wavenumber $k_{c}=V /\left(2 \nu_{s}\right)=k_{0} / 2$. The temperature of the helium bath determines $\alpha$ and the applied heat current determines $V$, so the Kelvin wave which grows exponentially faster and dominates all the other waves has wavelength $\lambda_{c}=4 \pi \nu_{s} / V$.

\section{The AC instability: second sound}

In second sound the quantity $V$ reverses sign during a cycle. This can be achieved by using Nuclepore transducers, which are oscillating membranes covered by holes which are so small (sub-micron size) that the viscous normal 


\section{Barenghi, Tsubota, Mitani and Araki}

fluid cannot move through them. In this way an oscillating counterflow velocity is set up and Eq. (6) becomes

$$
\frac{d A}{d t}=\alpha\left(k V_{0} \cos \left(\omega_{0} t\right)-\nu_{s} k^{2}\right) A
$$

Letting $t^{\prime}=\omega_{0} t$ and $\mu=\nu_{s} k / V_{0}$ the solution of Eq. (8) is

$$
A\left(t^{\prime}\right)=A(0) e^{f\left(t^{\prime}\right)}
$$

where

$$
f\left(t^{\prime}\right)=\frac{\alpha k V_{0}}{\omega_{0}}\left[\sin \left(t^{\prime}\right)-\mu t^{\prime}\right]
$$

The amplitude $A$ of the perturbation grows if $f>0$, but, since $\left|\sin \left(t^{\prime}\right)\right| \leq$ 1 , we have $f<0$ for sufficiently long times. We conclude that infinitesimal perturbations of any wavenumber $k$ decay exponentially for $t \rightarrow \infty$. However, although there are no instabilities for $t \rightarrow \infty$, for a limited initial time helical waves can actually increase their amplitude. This "transient growth" takes place in the time interval such that $\sin \left(t^{\prime}\right)>\mu t^{\prime}$ that is to say for

$$
t<\frac{V_{0}}{\nu_{s} k \omega_{0}}
$$

The effect can be significant (it can last for an appreciable time) at low second sound frequency $\left(\omega_{0}<<1\right)$, high second sound amplitude $\left(V_{0}>>1\right)$ and long wavelength $(k<<1)$, because $\nu_{s} \approx 10^{-3} \mathrm{~cm}^{2} / \mathrm{sec}<<1$ (taking

$a_{0} \approx 10^{-8} \mathrm{~cm}$ and $\Omega \approx 1 \mathrm{rad} / \mathrm{sec}$ ). Note that the longest wavelength depends on the size of the channel. The important point to notice is that a great range of wavenumbers can be affected by transient growth. Since mechanical and thermal perturbations are created all the time, if these perturbations grow before decaying to zero at $t \rightarrow \infty$, then each vortex line will have a certain amount of small amplitude Kelvin waves, depending on the particular set up of the experiment ( $\omega_{0}, V_{0}$ and the size of the channel) By attenuating the second sound which propagates in the direction along the vortices, these Kelvin waves may lead to think that there exists a longitudinal coefficient of mutual friction.

\section{The AC instability: oscillating counterflow}

Perhaps the simplest way to produce a time dependent heat flux $Q$ is to apply an alternating voltage to the resistor $R$ which generates the heat 


\section{Transient growth of Kelvin waves on quantized vortices}

current. Since $Q$ is proportional to the square of the applied voltage, Eq. (8) must be replaced by

$$
\frac{d A}{d t}=\alpha\left[k V_{0} \cos ^{2}\left(\omega_{0} t\right)-\nu_{s} k^{2}\right] A,
$$

Preceding as before we have

$$
\frac{d A}{d t^{\prime}}=\frac{\alpha k V_{0}}{\omega_{0}}\left[\cos ^{2}\left(t^{\prime}\right)-\mu\right] A,
$$

and the solution is

$$
A\left(t^{\prime}\right)=A(0) e^{f\left(t^{\prime}\right)}
$$

where

$$
f\left(t^{\prime}\right)=\frac{\alpha k V_{0}}{2 \omega_{0}}\left[(1-2 \mu) t^{\prime}+\frac{1}{2} \sin \left(2 t^{\prime}\right)\right],
$$

The perturbations grow if $f\left(t^{\prime}\right)>0$. The long term behaviour of $f\left(t^{\prime}\right)$ depends on the first term in Eq. (15) which increases with $t^{\prime}$, not on the second which is always less than unity. If the first term is positive $(1-2 \mu>0)$ then $A$ grows for $t \rightarrow \infty$; this happens for $\mu<1 / 2$, that is for $0<k<\frac{V_{0}}{2 \nu_{s}}$ which is the same condition as for the DC instability with $V_{0}$ replaced by $V_{0} / 2$. Transient growth is possible for a limited time even if $1-2 \mu<0$ provided that is for $t<1 /\left(2 \omega_{0}|1-2 \mu|\right)$.

\section{Conclusion}

We have shown that there is an "AC" version of the "DC" Donnelly Glaberson instability, and that can it be realized in a controlled way in the laboratory, either using second sound Nuclepore transducers or an oscillating counterflow. We have determined the boundaries between the stable and the unstable regions and found that in the linearly stable regions, although each individual perturbation decays as $t \rightarrow \infty$, a state of transient growth is possible which depends on the frequency and the amplitude of the second sound wave and on the length of the channel. This state of transient growth of Kelvin waves would produce attenuation of second sound in the direction parallel to the quantised vortices and would be interpreted as evidence for a longitudinal component of mutual friction. The possibility of the existence of a longitudinal friction coefficient (called $B^{\prime \prime}, B_{z}$ or $\gamma$ ) was considered in the earlier helium II literature ${ }^{11}$. Experiments ${ }^{12}$ concluded that $B^{\prime \prime}$, if it exists at all, is very small (at the most 500 times smaller than $B$ ), is not reproducible and may depend on the amplitude of the second sound wave 


\section{Barenghi, Tsubota, Mitani and Araki}

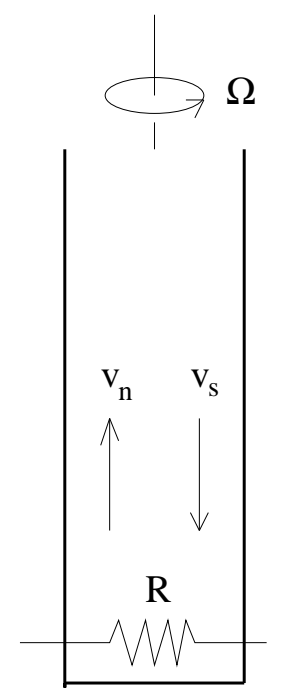

Fig. 1. Rotating counterflow channel configuration.

used to measure it, which is consistent with our finding. We plan to continue this investigation using numerical simulations.

C.F.B. is grateful to the Royal Society for supporting this study.

\section{REFERENCES}

1. D.C. Samuels and D. Kivotides, Phys. Rev. Lett. 83, 5306 (1999).

2. D. K. Cheng, M.W. Cromar and R.J. Donnelly, Phys. Rev. Lett. 31, 433 (1973).

3. R.M. Ostermeier and W.I. Glaberson, J. Low Temp. Phys. 21, 191 (1975);

4. M. Tsubota, T. Araki and C.F. Barenghi, Phys. Rev. Lett. 90, 205301 (2003).

5. L. Skrbek, J.J. Niemela and R.J. Donnelly, Phys. Rev. Lett. 85, 2973 (2000).

6. J. Maurer and P. Tabeling, Europhys. Lett. 43, 29 (1998).

7. C.F. Barenghi, D.C. Samuels, G.H. Bauer and R.J. Donnelly, Phys. Fluids 9, 2631 (1997).

8. C.F. Barenghi, S. Hulton and D.C. Samuels, Phys. Rev. Lett. 89275301 (2002).

9. P.G. Saffman Vortex Dynamics, Cambridge University Press, (1992).

10. K.W. Schwarz, Phys. Rev. B 38, 2398 (1988).

11. I.M. Khalatnikov, An Introduction To The Theory Of Superfluidity, W.A. Benjamin Inc. (1965).

12. H.A. Snyder, Phys. Fluids 6, 755 (1963). 